\title{
Pour une poétique du nom de personnage
}

\author{
SAMUEL BIDAUD
}

\begin{abstract}
Prolegomena to a poetics of the character's name. We propose in this article a poetics of the character's name. The character's name can be studied from an autonomous point of view as well as from a structural point of view. From an autonomous point of view, at first, we show that the character's name especially reflects a personal, a social, a physical, a generic, a geographical, an autobiographical or a referential characteristic of the character. We also focus on two particular cases, the case in which the name of the character is incomplete and the case in which the identity of the character is changing. The structural point of view, on the contrary, consists in studying the names of the characters of a same work by comparing them to each other. We focus in this way on the thematic role of the characters' names, and on the case in which some phonetic features are recurrent in the names of several characters and must therefore be interpreted. We eventually mention the problem of the translation of the characters' names, focusing on two situations: the situation in which the character's name has no obvious meaning, and is not really translated, and the situation in which the character's name has a real meaning, and is either translated literally or adapted.
\end{abstract}

Keywords: poetics; characters' names; general literature; translation

\section{Introduction}

La linguistique contemporaine, notamment par les recherches qui portent sur le phonosymbolisme, remet de plus en plus en question la théorie de l'arbitraire du signe, qui n'avait d'ailleurs jamais entièrement prévalu si l'on considère l'histoire des idées linguistiques ${ }^{1}$. Or, s'il est un domaine où le signe échappe complètement à l'arbitraire, c'est celui du nom de personnage. L'auteur, en effet, ne nomme pas ses personnages par hasard, et les noms qu'il donne à ces derniers sont, à des degrés variés, motivés. Nous envisageons ici de proposer une poétique du nom de personnage. Plusieurs études de très bonne qualité se sont, bien sûr, déjà penchées sur le sujet, mais aucune de façon vraiment

Ainsi trouve-t-on l'idée d'une motivation du signe aussi bien chez Otto Jespersen que chez Maurice Grammont ou Edward Sapir, pour citer quelques noms importants de la linguistique.

DOI: http://dx.doi.org/10.12697/IL.2016.21.1.10 
BIDAUD

systématique (voir toutefois Léonard et Nardout-Lafarge 1996, et Erman 2006 : 33-50). Il existe, et ce sera la distinction de départ qui structurera notre article, deux façons d'étudier les noms de personnages : ces derniers peuvent être étudiés de façon autonome, ou alors de façon «structurale », c'est-à-dire les uns par rapport aux autres.

Les noms, considérés d'un point de vue individuel, peuvent tout d'abord refléter une caractéristique du personnage. Il peut s'agir d'une caractéristique morale, sociale, physique, générique, etc. ; nous nous efforcerons, d'une façon nécessairement non exhaustive, de proposer une typologie des principales significations que peut revêtir le nom, en nous penchant pour finir sur deux cas particuliers, le cas où l'identité du personnage est incomplète, et le cas où le personnage change de nom.

Nous étudierons ensuite les noms de personnages de façon structurale. Les noms de personnages, en effet, forment un système à l'intérieur duquel ils occupent une fonction les uns relativement aux autres. Nous montrerons d'abord que les noms de personnages peuvent être regroupés par rapport à la thématique qu'ils reflètent. Tel est le cas, par exemple, dans À la recherche $d u$ temps perdu, où les noms de personnages sont liés à la thématique de l'homosexualité et de la bisexualité. Il arrive également que certains phonèmes ou certaines syllabes, que nous nommerons « formants », reviennent dans les noms de plusieurs personnages de façon prédominante, ce qui ne peut être dû au hasard et doit être interprété, comme nous le verrons là encore essentiellement à partir d'À la recherche du temps perdu.

Enfin, nous nous pencherons rapidement, dans une dernière partie, sur le problème de la traduction des noms de personnages.

Précisons que si le nom de personnage est généralement étudié à partir de la littérature, nous citerons également dans cette étude des exemples empruntés au cinéma, puisqu'une poétique du nom de personnage englobe par nature littérature et cinéma ${ }^{2}$.

2 Nous avons récemment consacré deux articles aux noms des personnages d'À la recherche du temps perdu et des Aventures de Tintin, dont le premier a paru dans le Bulletin Marcel Proust (Bidaud 2015) et dont le second devrait normalement paraitre dans l'Irish Journal of French Studies (Bidaud, à paraître) ; aussi les exemples que nous donnons concernant ces œuvres sont-ils largement empruntés à ces articles. 
Pour une poétique du nom de personnage

Fonction caractérisante du nom

On peut tout d'abord parler d'une fonction « caractérisante » des noms de personnages. Nous distinguerons plusieurs cas dans cette perspective.

\section{A. Le nom renvoie à un trait de la personnalité du personnage}

Le nom peut refléter une caractéristique de la personnalité de celui qui le porte. Il peut s'agir d'une qualité : Charles Swann, dans À la recherche du temps perdu, a un nom de famille qui renvoie à l'élégance du personnage, puisque « swann » signifie cygne en anglais et en allemand; or on sait que l'amant d'Odette, familier du prince de Galles, se caractérise par sa distinction. Virginie, dans Paul et Virginie, a un prénom qui renvoie à l'innocence, et le roman de Bernardin de Saint-Pierre se déroule effectivement dans un univers innocent, à l'écart de la civilisation. Il arrive également que ce soit un surnom qui renvoie à une qualité du personnage : Tintin est ainsi surnommé « Cour pur » par les moines qui le recueillent dans Tintin au Tibet. Mais le nom peut aussi renvoyer à un défaut : le nom du professeur Isak Borg, dans Les Fraises sauvages de Bergman, reflète, comme cela a été remarqué plusieurs fois, le caractère glacial du personnage, dont le film raconte l'humanisation progressive (on entend en effet « is », c'est-à-dire « glace » en suédois, dans Isak).

Si dans les cas que nous avons mentionnés la qualité ou le défaut ne sont pas explicitement formulés et qu'ils sollicitent un effort d'interprétation, le nom peut également parfois correspondre littéralement à une qualité ou un défaut du personnage : c'est le cas pour le Candide de Voltaire.

Le nom peut bien sûr renvoyer à la personnalité du personnage sans renvoyer à un trait de caractère à proprement parler. Dans Laurence anyways, le film de Xavier Dolan, le personnage principal, Laurence, change de sexe au cours du film ; or son prénom, qui est un prénom masculin en anglais, est un prénom féminin en français, ce qui reflète la transsexualité du personnage.

\section{B. Le nom renvoie à une fonction sociale du personnage}

Par « fonction sociale » du nom nous comprenons d'une part les cas où le nom renvoie à l'activité du personnage, d'autre part les cas où il renvoie à son rang.

Le nom peut renvoyer à l'activité du personnage. Il est fréquent que le nom renvoie à l'activité du personnage par association : dans Le Malade imaginaire de Molière, monsieur Purgon a un nom scatologique qui renvoie à sa profession de médecin et au fait qu'il administre force lavements à Argan. Le nom du personnage peut également renvoyer à sa profession par connotation : 
BIDAUD

tel est le cas pour Igor Wagner, le pianiste de la Castafiore dans Tintin, dont le nom renvoie au musicien allemand et connote l'univers de la musique.

À côté de son activité professionnelle, le personnage peut être désigné par son rang. On relèvera dans un premier temps le cas des appellations directes : le Cavaliere de la Locandiera de Goldoni n'est connu que sous cette appellation. Mais le rang du personnage peut également être contenu dans la sémantique de son nom. Dans $\grave{A}$ la recherche $d u$ temps perdu, plusieurs personnages portent un nom dans lequel on entend leur noblesse : le duc de Châtellerault, où l'on entend « chastel », le comte d'Argencourt, où l'on entend « argent », et le prince d'Agrigente et le marquis de Beausergent, dans les noms desquels résonne également l'écho de « argent ». Dans la Recherche, l'opposition sociale se reflète également dans les finales des noms, comme l'a bien vu Roland Barthes, qui note la présence d'« un large système onomastique, articulé sur l'opposition de l'aristocratie et de la roture d'une part, et sur celle des longues à finales muettes (finales pourvues en quelque sorte d'une longue traîne) et des brèves abruptes d'autre part : d'un côté le paradigme des Guermantes, Laumes, Agrigente, de l'autre celui des Verdurin, Morel, Jupien, Legrandin, Sazerat, Cottard, Brichot, etc. » (Barthes 1972 : 128).

\section{Le nom renvoie à une caractéristique physique du personnage}

Le nom peut renvoyer à une caractéristique physique du personnage. Gargantua est nommé ainsi par Grandgousier parce qu'il demande du vin à sa naissance : «Que grande tu l'as ! ( (sous-entendu la gorge), s'exclame alors Grandgousier. Mais dans la majorité des cas c'est le surnom qui reflète une caractéristique physique du personnage : on pensera par exemple à Poil-decarotte.

\section{Le nom renvoie à un type de personnage}

Le nom peut également rattacher le personnage à un «type » de personnage. Sganarelle, Arlequin, Valère ou Lisette, par exemple, sont des noms typiques de personnages de comédie, que l'on retrouve de Molière à Marivaux. On peut à l'intérieur de ces catégories préciser certaines distinctions : Sganarelle, Lisette et Arlequin sont plutôt des noms de domestiques, alors que Valère, Clitandre ou Elise sont plutôt des noms d'amants bourgeois. Le nom a donc une fonction générique, dans le sens où il peut être typique de certains genres : on imagine mal, par exemple, une tragédie dont les héros se nommeraient Sganarelle et Lisette, et, inversement, on imagine mal des servantes de comédies qui s'appelleraient Phèdre ou Andromaque. 


\section{E. Le nom renvoie à l'origine géographique du personnage}

Le nom peut renvoyer à l'origine géographique du personnage. Parfois le personnage n'a pas d'autre identité que la mention de son origine : le maharadjah de Rawajpoutalah n'est nommé que par son titre et la région où il règne dans Les Cigares du pharaon. Mais l'origine géographique peut également être contenue dans le nom du personnage, et n'est pas obligatoirement exprimée de façon périphrastique. Dans À la recherche du temps perdu, Françoise représente la « francité », à la fois par son langage qui a gardé de vieilles expressions et par la terre de Combray de laquelle elle fait partie (ce Combray «symbole de la terre de France d'où le génie s'exhale », comme l'écrit Sylvie Pierron (1999 : 56) dans un article qu'elle consacre à la langue de Françoise) ; ce n'est donc pas par hasard si l'on entend « France » dans Françoise. L'origine géographique peut également être ajoutée au nom du personnage comme une forme de surnom. Dans Le Mariage de Mlle Beulemans, la pièce de Wicheler et Fonson qui eut un énorme succès aussi bien à Bruxelles qu'à Paris au début du siècle dernier, Albert, le jeune Français envoyé par son père en Belgique s'initier au commerce belge auprès de monsieur Beulemans, est surnommé « le fransquillon » ou « monsieur Albert de Paris » par monsieur Beulemans et Séraphin Meulemeester, qui lui reprochent son langage affecté et les airs qu'il se donne.

\section{F. Le nom a une valeur autobiographique}

Le nom de personnage peut avoir une valeur autobiographique. Par « valeur autobiographique » nous entendons les cas où le nom renvoie à l'auteur, ce qui déborde le cadre de l'autobiographie à strictement parler. Nous distinguerons les noms de personnages à valeur autobiographique directe des noms de personnages à valeur autobiographique indirecte.

Les noms de personnages à valeur autobiographique directe renvoient directement à l'auteur, dont ils n'altèrent pas l'identité. Le cas le plus évident est celui de l'autobiographie : auteur et personnage ne font alors qu'un, par exemple Jean-Jacques Rousseau et Jean-Jacques dans Les Confessions. Mais l'autobiographie n'est pas le seul cas où le nom du personnage renvoie directement à l'auteur : le personnage de Molière, par exemple, dans L'Impromptu de Versailles, renvoie également à l'auteur « Molière », et à la fin de Niebla (Brouillard), le roman de Miguel de Unamuno, le personnage principal vient implorer Unamuno, qui apparait comme personnage (personnage-auteur pourrait-on dire), de le laisser vivre.

Les noms de personnages à valeur autobiographique indirecte renvoient au contraire à l'auteur en altérant son identité, laquelle se laisse supposer sans être posée. Le personnage d'Antoine Doinel, dans le cycle de François Truffaut 
BIDAUD

(Les 400 coups, Antoine et Colette, Baisers volés, Domicile conjugal et L'Amour en fuite), renvoie à Truffaut. Ce dernier confiait en effet qu'Antoine Doinel était moitié lui-même et moitié Jean-Pierre Léaud ; or le rapport autobiographique se retrouve ici dans l'onomastique : entre Antoine et François, il y a deux phonèmes en commun, « an » et « oi », dans la même position, c'est-à-dire respectivement dans la première et la seconde syllabe, ce que l'on ne retrouve dans aucun autre prénom français. Toujours au cinéma, on remarquera, après d'autres, que le professeur Isak Borg, dans Les Fraises sauvages, a les mêmes initiales qu'Ingmar Bergman.

L'auteur peut jouer de la valeur autobiographique du nom du personnage. On notera ici le cas particulier d'À la recherche $d u$ temps perdu, qui n'est certes pas autobiographique mais où l'auteur donne au narrateur son propre prénom, et cela sous forme hypothétique : «Dès qu'elle retrouvait la parole elle disait : "Mon" ou "Mon chéri" suivis l'un ou l'autre de mon nom de baptême, ce qui, en donnant au narrateur le même nom qu'à l'auteur de ce livre, eût fait : "Mon Marcel”, "Mon chéri Marcel” » (Proust 1999 : 1658).

\section{G. Le nom a une valeur référentielle}

Le nom de personnage peut, plus largement, avoir une valeur référentielle et renvoyer à une personne réelle, là encore de façon directe ou indirecte.

Le nom peut renvoyer à une personne réelle de façon directe, c'est-àdire sans altérer son identité. Nombreux sont les personnages historiques qui apparaissent dans des œuvres littéraires. À la fin de L'Âge de raison, par exemple, le premier tome des Chemins de la liberté, Sartre décrit le retour de Daladier à Paris.

Mais le nom du personnage peut également renvoyer à une personne réelle en altérant son identité. On pourra citer ici le cas du personnage de Jean-Sol Partre, dans L'Écume des jours de Boris Vian, derrière lequel on reconnaît sans difficulté Jean-Paul Sartre.

\section{H. L'identité du personnage est incomplète}

Il arrive que l'identité du personnage soit incomplète. Nombreux sont les personnages dont on ne connaît que le prénom ou que le nom : on ne connaît pas le nom de famille de Tintin ou le prénom de Dupond et Dupont dans Tintin, et rien ne dit même très explicitement que Tintin soit un prénom, puisque Tintin se fait parfois appeler «Monsieur Tintin ». Mais par «identité incomplète $\gg$ on peut également comprendre le cas où ni le prénom ni le nom du narrateur ne sont connus. Ainsi le personnage est fréquemment désigné par une simple lettre, comme K dans Amerika et dans Le Procès de Kafka, avec un $\ll \mathrm{K} \gg$ qui fait d'ailleurs écho au $\ll \mathrm{K} \gg$ de Kafka. Le fait de ne désigner le 
personnage que par une lettre permet ici de refléter un univers angoissant où l'individu n'a plus de repères. Dans La Jalousie de Robbe-Grillet, A..., la femme du narrateur, est également appelée par une seule lettre suivie de points de suspension, ce qui reflète le caractère obsessionnel de la jalousie du narrateur qui ressasse sans cesse cette même initiale.

\section{L'identité du personnage est changeante}

Il arrive que l'identité du personnage soit instable, et que le personnage change de nom. Ce changement de nom a généralement une fonction narrative : dans Tintin, le docteur Müller, rencontré dans L'T̂le noire, se fait appeler professeur Smith dans Au pays de l'or noir, ce qui brouille les pistes quant à son identité ; de même, le colonel Sponsz de L'Affaire Tournesol se fait appeler colonel Esponja dans Tintin et les Picaros. Le changement de nom peut également avoir une fonction thématique : À la recherche du temps perdu développe l'idée que nous ne voyons des êtres qui nous entourent que des aspects changeants, ce qui se reflète dans le changement de nom d'un personnage comme Odette, laquelle est d'abord connue du lecteur comme « la dame en rose », puis comme Odette, et enfin comme Miss Sacripant, nom sous lequel elle a posé pour Elstir lorsqu'elle était plus jeune.

On pourra, toujours dans la perspective du thème de l'identité changeante, distinguer les noms attribués par l'auteur (les noms qui ne dépendent pas des personnages appartenant à l'univers fictionnel) et les noms qui proviennent de l'univers fictionnel lui-même. Dans la majorité des cas, le nom donné au personnage par l'auteur coïncide avec celui que lui donnent les autres personnages : Charles Swann, par exemple, est bien connu comme Charles Swann par les autres personnages d'À la recherche $d u$ temps perdu. Mais dans certains cas, il peut y avoir un conflit quant au nom. On pourra citer ici l'exemple de Pierrot le fou, le film de Jean-Luc Godard, où le personnage principal ne cesse de répéter, tout au long du film, « Je m’appelle Ferdinand » à Marianne, qui ne cesse de l'appeler Pierrot, nom qui donne finalement son titre au film.

\section{Le nom comme système}

Nous nous sommes jusqu'à maintenant intéressé au nom de personnage de façon « autonome », c'est-à-dire que nous avons étudié ce dernier pour lui-même et non par rapport aux autres noms avec lesquels il entre en résonance. Or, dans une œuvre, littéraire ou cinématographique, les noms des personnages se font également écho les uns aux autres, et peuvent 
être étudiés de façon «structurale ». On peut, d'abord, se demander si les noms des personnages peuvent être liés à la thématique de l'œuvre : nous montrerons qu'il en va ainsi dans $\grave{A}$ la recherche $d u$ temps perdu. Nous verrons dans un deuxième temps qu'il est possible d'isoler, à l'intérieur des noms des personnages d'une même œuvre, des phonèmes ou des syllabes récurrents que nous nommerons « formants », dont la fréquence est telle qu'elle ne peut être due au hasard, et qui ont de ce fait une signification qu'il convient de dégager.

\section{A. La fonction thématique du nom}

Les noms de personnages peuvent avoir une fonction thématique, c'est-à-dire être liés à une thématique de l'œuvre, comme c'est le cas dans À la recherche $d u$ temps perdu avec la thématique de l'homosexualité et de la bisexualité. En effet, la thématique de l'homosexualité est omniprésente dans $A$ la recherche $d u$ temps perdu, où les personnages se révèlent progressivement homosexuels ou bisexuels ; or cette thématique de l'homo- et de la bisexualité se reflète dans l'onomastique.

On a souvent remarqué, d'abord, que les jeunes filles en fleurs aimées du narrateur portaient des prénoms féminins qui étaient la féminisation de prénoms masculins : Albertine, Andrée, Gilberte, - cette dernière étant la seule à ne pas avoir eu de relation avec des femmes. Les noms d'Albertine et d'Andrée reflètent donc l'ambivalence de leur sexualité, puisqu'elles sont, comme leur nom, tournées aussi bien vers les hommes que vers les femmes.

Mais plusieurs autres personnages ont des noms qui renvoient également à l'inversion. Le baron de Charlus a comme prénom Palamède, prénom qui renvoie à la Grèce et qui connote l'homosexualité dans le cas du baron. Quant au nom Charlus, le « us » final vient comme nier la majesté de « Charles » et montre que le baron n'est pas un «vrai »Charles, c'est-à-dire que le «us » nie la virilité que le baron affiche et affecte. Il en va de même pour son amant Charlie Morel, où le « ie » final de Charlie vient là encore nier le caractère masculin du musicien. Au baron de Charlus et à Charlie Morel, qui ne sont pas de vrais Charles, on opposera Charles Swann, qui est au contraire grand amateur de femmes.

L'inversion se retrouve également dans le nom de Jupien, qui arrange les rendez-vous du baron : on entend « jupe » dans Jupien, ce qui renvoie à la métaphore du vêtement féminin et de la couture, liée dans la Recherche à l'inversion (on sait par exemple que le baron de Charlus est particulièrement amateur de vêtements féminins, et qu'il est surnommé « la Couturière »). On retrouve également le mot « robe » dans le prénom du neveu du baron de Charlus, Robert de Saint-Loup, lequel se révèle lui aussi homosexuel à la fin du roman. 
Pour une poétique du nom de personnage

Les noms des personnages de la Recherche que nous avons commentés, on le voit, contiennent donc en eux la thématique de l'inversion.

\section{B. Les formants}

Il arrive que certains phonèmes ou certaines syllabes se retrouvent de façon récurrente dans les noms de plusieurs personnages d'une même œuvre. Nous proposons d'appeler « formants » ces phonèmes et ces syllabes, qui ont bien sûr une valeur signifiante et doivent être interprétés.

Parfois le formant est constitué d'un seul phonème : dans La Coscienza di Zeno (La Conscience de Zeno) d'Italo Svevo, les quatre filles du beau-père de Zeno, qui fait une demande en mariage à trois d'entre elles, portent toutes un prénom qui commence par la première lettre de l'alphabet, A (Ada, Alberta, Augusta et Anna), et qui s'oppose, symboliquement, à celui de Zeno, qui commence au contraire par la dernière lettre de l'alphabet, $\mathrm{Z}$, comme les critiques n'ont pas manqué de le noter.

Le formant peut également être constitué de plusieurs phonèmes. On retrouve par exemple le formant /ber/ dans les noms de plusieurs personnages d'À la recherche du temps perdu: Gilberte, Albertine, Robert de Saint-Loup, la Berma et Bergotte. Alain Buisine note ainsi :

Maintes études critiques ont déjà remarqué que la dyade consonantique $b r$ insiste dans toute l'onomastique de la Recherche ( $b r$ souvent varié et nuancé en $p r$ ou en $v r$ ou même en $g r$ ). Incontestablement le noyau de cette diffusion semble bien être constitué par la syllabe BER statistiquement la plus fréquente : Gilberte et Albertine, Bergotte (Bergson) et la Berma (Sarah Bernhardt), toute la série des noms mérovingiens (Childebert, Dagobert, Théodebert, Sigebert, Gilbert le Mauvais qui fut d'abord Albert, puis Fulbert), maints noms de personnes et de lieux, Robert de Saint-Loup, Hubert Robert, Vaugoubert, Alberti, Nissim Bernard, Berthe, Gibergue, Imbert, Froberville, Berneville, Berry-au-Bac, Questembert, Thiberzy, etc. (Buisine 1984 : 61-62)

Alain Buisine propose une interprétation psychanalytique de la prédominance $\mathrm{du}$ formant /ber/ : ce formant se retrouve dans le nom de la grand-mère maternelle de Proust, Adèle Berncastel, et dès lors le formant /ber/ apparaît comme un écho du nom maternel.

Michel Erman identifie pour sa part un formant /er/, « commun aux noms des femmes aimées par le narrateur : Gilberte, Guermantes, Stermaria, Albertine $\gg$ (Erman $2006: 48)$.

Nous pensons que le formant /er/ peut également recevoir une interprétation plus large. Notons qu'on le retrouve dans les noms de très 
nombreux personnages de la Recherche : Gilberte, Guermantes, Stermaria et Albertine effectivement, mais également, parmi les personnages principaux, les Verdurin, Robert de Saint-Loup, Bergotte, la Berma, Albert Bloch et les Cambremer, et, parmi les personnages secondaires, le marquis de Beausergent, Nissim Bernard, Bernier (le valet de Charlus), Berthes (l'amie d'Albertine), les Chaussepierre, le marquis de Féterne, les Howsler (le cocher et le valet de pied des Verdurin), Mme Imbert à Combray, Joseph Périgot (le valet qui écrit à sa famille en recopiant des passages de livres), le docteur Percepied, $M$. Pierre (l'historien de la Fronde), la marquise de la Pommelière, la princesse Sherbatoff, M. Vallenères (l'archiviste des lettres de Mme de Villeparisis), le marquis de Vaugoubert, et le duc de Wurtemberg. Ajoutons que le formant /er/ se retrouve de façon inversée dans les noms de plusieurs personnages importants : Andrée, Saint-Loup en Bray et Morel, si on inverse les lettres du « rée » de Andrée, du « ray » de Bray et du « rel » de Morel.

Le formant /er/ renvoie d'après nous à l'«er-reur » qui prédomine dans la Recherche, où les personnages ne cessent de se révéler autre chose que ce que le narrateur pensait qu'ils étaient.

Le formant peut enfin correspondre à un nom complet, comme c'est le cas avec le nom «Charles » dans la Recherche. Comme le note Michel Erman à propos de Charlus :

Le nom Charlus fait partie d'un paradigme dans lequel on retrouve Charles Morel, Saint-Loup (dans un premier état du roman, celui-ci se prénommait Charles et non pas Robert), la maîtresse de ce dernier, Rachel (anagramme de Charles) et encore Charles Swann. Le signifiant Charl(us) semble bien relever d'un ensemble d'effets connotatifs. Il fonctionne, en premier lieu, par association : Swann et Saint-Loup ont, tous deux, été trompés par la personne qu' ils aimaient (Odette et Rachel) comme Charlus le sera par Morel, de plus le narrateur se demandera si Rachel n'a pas été à l'origine du changement d'ordre sexuel qui affectera Saint-Loup. Le signifiant fonctionne aussi en miroir, c'est-à-dire en image inversée : Morel et Rachel semblent être les supports des projections affectives de leurs amants respectifs. (Erman $2006: 49$ )

Le formant, on le voit, peut donc en fin de compte être constitué aussi bien par un phonème unique, que par une syllabe ou un nom, et se caractérise par le fait d'être récurrent et significatif.

\section{La question de la traduction du nom de personnage}

Il est bien sûr impossible de traiter, en quelques paragraphes, de la question de la traduction des noms de personnages, mais il nous a paru opportun, en 
guise d'ouverture, d'aborder rapidement le sujet, qui fait pleinement partie de la problématique qui nous occupe.

Le problème est le suivant : comment rendre dans une langue étrangère un nom de personnage motivé ?

La question de la motivation thématique et structurale du nom doit dès le départ être écartée : il ne saurait être question, pour le traducteur, de découvrir le système onomastique d'À la recherche $d u$ temps perdu, dont ni l'auteur (Proust) ni le lecteur ne sont conscients, et que seule l'analyse permet de mettre au jour.

Les noms de personnages ne pourront être traduits que lorsque leur motivation est évidente. Dans la plupart des cas donc, les noms resteront les mêmes dans le texte d'origine et dans la traduction : Charles Swann ne sera pas traduit et restera identique dans la langue de départ, le français, et la langue d'arrivée, le suédois, l'anglais, le norvégien ou le tchèque. Les noms peuvent bien sûr parfois être légèrement adaptés graphiquement ou phonétiquement : Tintín en espagnol (adaptation graphique), Tintim en portugais (adaptation phonétique). Parfois également le nom est traduit par son équivalent dans la langue d'arrivée : Françoise, dans À la recherche du temps perdu, devient Františka en tchèque.

Dans le cas où le nom est motivé de façon évidente, et fait cette fois l'objet d'une « vraie » traduction, il s'agit pour le traducteur ou de traduire littéralement le nom du personnage, ou de trouver un équivalent dans la langue d'arrivée.

Le premier cas, celui de la traduction littérale, se présente par exemple avec Pippi Långstrump, l'héroïne des livres d'Astrid Lindgren. Pippi Långstrump, c'est-à-dire littéralement, en suédois, «Pippi longues chaussettes », est ainsi traduit par Pippi Calzelunghe en italien, donc, là encore littéralement, par « Pippi longues chaussettes ». Une telle traduction, en revanche, serait particulièrement lourde en français.

Ce qui nous amène au second cas : celui où le traducteur « adapte »le nom de personnage. Le français adapte ainsi le nom de Pippi Långstrump, qui devient Fifi Brindacier. Le nom suédois est motivé et renvoie à une caractéristique physique du personnage (qui porte des chaussettes longues), et le nom français est également motivé, et renvoie lui aussi à une caractéristique du personnage, mais il ne s'agit plus de la même caractéristique qu'en suédois : Fifi, en effet, est une petite fille qui se caractérise par sa force ; c'est donc, littéralement, un « brin d'acier ». La traduction française, on le notera au passage, est particulièrement réussie.

Le traducteur peut, plus rarement, retenir une propriété phonétique du nom. Dupond et Dupont, dans Tintin, sont presque identiques physiquement 
BIDAUD

(seule leur moustache permet de les différencier), et ont des noms qui sont phonétiquement similaires et ne se distinguent que par l'orthographe finale. Les traducteurs vont alors garder le même principe de deux noms presque identiques dans la langue d'arrivée : Kadlec et Tkadlec en tchèque, Hernández et Fernández en espagnol, etc. Le nom du personnage de Tryphon Tournesol, toujours dans Les Aventures de Tintin, est formé sur un redoublement des initiales, ce qui crée un effet phonétique particulier ; aussi les langues d'arrivée garderont-elles là encore parfois ce principe : Tryphon Tournesol devient Karl Kalkyl en suédois, Balduin Bienlein en allemand, etc.

On peut donc envisager plusieurs cas en ce qui concerne la traduction du nom de personnage. Dans le premier cas, la motivation du nom n'est pas transparente, et le nom ou n'est pas traduit, ou fait l'objet d'une légère adaptation au système phonétique/graphique/onomastique de la langue d'arrivée (Tintín en espagnol, Tintim en portugais, Františka pour Françoise dans la version tchèque d'À la recherche $d u$ temps perdu). Dans le second cas, le nom a une motivation évidente dans la langue d'origine. Le traducteur peut alors ou traduire littéralement le nom (Pippi Långstrump qui devient Pippi Calzelunghe en italien), ou sélectionner une caractéristique du personnage qui n'est pas présente dans la langue de départ pour la faire ressortir dans la langue d'arrivée (Pippi Långstrump toujours, qui devient Fifi Brindacier en français).

\section{Conclusion}

Les noms de personnages, nous l'avons vu pour commencer, peuvent refléter une caractéristique de ces derniers : caractéristique morale, sociale, physique, générique ou géographique essentiellement. Les noms peuvent également être chargés d'une valeur autobiographique ou référentielle, et être incomplets ou changeants. Un tel point de vue, qui envisage le nom de personnage pour lui-même, est bien sûr légitime. Mais à un niveau supérieur, les noms de personnages forment un système, et sont liés les uns aux autres. Nous avons commencé par voir que les noms pouvaient refléter une thématique de l'œuvre : tel est le cas avec la thématique de l'inversion dans $\grave{A}$ la recherche $d u$ temps perdu. Nous avons montré, ensuite, que certains éléments phoniques, que nous avons appelés « formants », revenaient parfois dans les noms de plusieurs personnages d'une même œuvre, et que cette récurrence n'était bien sûr pas due au hasard et pouvait être interprétée thématiquement. Nous nous sommes enfin penché sur la question de la traduction des noms de personnages, et avons envisagé les cas où le nom n'a pas de motivation évidente dans la langue de départ et n'est donc pas traduit dans la langue d'arrivée, et 
les cas où le nom a une motivation évidente dans la langue de départ, et est soit traduit, soit fait l'objet d'une adaptation dans la langue d'arrivée, qui conserve un principe de motivation mais fait ressortir une autre caractéristique du personnage que la langue de départ.

Qu'on les étudie de façon autonome, comme formant un système ou du point de vue de leur traduction, les noms de personnages sont en tout cas toujours motivés à quelque niveau ; ils représentent, avec la poésie, le seul cas où l'auteur parvienne à se dégager de l'arbitraire du signe.

\author{
Samuel Bidaud \\ bidaudsamuel@gmail.com \\ Univerzita Palackého v Olomouci \\ Neředín 4,804 \\ U Letiště 847 \\ 77900 Olomouc \\ ČESKA REPUBLIKA / RÉPUBLIQUE TCHÈQUE
}

\title{
Bibliographie
}

Barthes, R. 1972. Proust et les noms. - R. Barthes, Le Degré zéro de l'écriture, suivi de Nouveaux essais critiques. Paris : Seuil, 122-156.

Bidaud, S. 2015. Les noms de personnages dans $\grave{A}$ la recherche du temps perdu. Bulletin Marcel Proust, 65, 31-38.

Bidaud, S. [à paraître]. À propos des noms de personnages de Tintin. Note d'onomastique littéraire. - Irish Journal of French Studies.

Buisine, A. 1984. Matronymies. - Littérature, 54, 54-78.

Erman, M. 2006. Poétique du personnage de roman. Paris : Ellipses.

Léonard, M., Nardout-Lafarge, É., éds. 1996. Le texte et le nom. Montréal : XYZ Éditeur.

Pierron, S. 1999. La langue Françoise dans À la recherche du temps perdu de Marcel Proust. - Littérature, 116, 47-58.

Proust, M. 1999. La Prisonnière. - M. Proust, À la recherche du temps perdu. Paris : Gallimard, 1607-1917. 